\title{
New Radio Numerology and Waveform Evaluation for Satellite Integration into 5G Terrestrial Network
}

\author{
Arunprakash Jayaprakash*, Barry G. Evans*, Pei Xiao*, Adegbenga B. Awoseyila* and Yingnan Zhang ${ }^{\dagger}$ \\ ${ }^{*}$ Home of 5G Innovation Centre, Institute for Communication Systems, University of Surrey, Surrey GU2 7XH, U.K \\ *Email: \{a.jayaprakash, p.xiao, b.evans, a.awoseyila\}@ surrey.ac.uk \\ ${ }^{\dagger}$ CAST Xi’an, China, Email: 66972515@qq.com
}

\begin{abstract}
This paper analyses the New Radio (NR) air interface waveforms and numerologies in the context of current activities and studies of 3 GPP related to the feasibility and standardisation of necessary adaptations for the 5G NR to support integrated-satellite-terrestrial networks with low earth orbit (LEO) satellites. Frequency-localized orthogonal frequency division multiplexing (OFDM)-based candidate waveforms are recommended by $3 \mathrm{GPP}$ as the waveforms for the NR in order to preserve the advantages of OFDM as well as maintain backward compatibility. 5G New Radio enables diverse service support, efficient synchronization and channel adaptability using a multinumerology concept, which defines a family of parameters of the parent waveform, that are related to each other by scaling. The major design challenges in the LEO satellite scenario are power limited link budget and high Doppler effects which can be addressed by choosing waveforms with small peak to average power ratio (PAPR) and sub-carrier bandwidth adaptation respectively. Hence, the selection of the right waveform and numerology is of prime relevance for the proper adaptation of 5G NR for LEO satellite terrestrial integration. The performance evaluation of the new air interface waveforms, with different numerologies, are carried out under the effect of carrier frequency offset (CFO), multipath effects, non-linearity, phase noise and additive white Gaussian noise (AWGN).
\end{abstract}

Index Terms-5G, numerology, satellite-eMBB, NTN, f-OFDM, W-OFDM, TWTA

\section{INTRODUCTION}

Satellite communication systems, with the emergence of high throughput satellites (HTS) and non-geo-stationary-orbit satellite constellation, can complement the terrestrial networks to meet the comprehensive and diverse service requirements of 5G. The initial studies on possible role of satellites with terrestrial mobile radio communications was initiated by $3 \mathrm{GPP}$ in Release 14 [1], in the form of a use-case-scenario study. In Release 15, 3GPP has completed a study which focused on definition of non terrestrial networks (NTN) deployment scenarios, adaptation of channel models for NTN and identification of key impact areas on the new-radio (NR) interface that need further evaluations [2]. The on-going follow-up study item by $3 \mathrm{GPP}$ in Release 16, related to NTN standardisation activity, identifies solutions for NR to support NTN [3]. In the 3GPP plenary meeting held at Shenzen/China in March 2019, preliminary views on NTN activities in Release-17 were discussed [4] and in the recent 3GPP meeting, it was decided to include 5G NR over NTN as a part of Release17. Performance assessment of NR in low earth orbit (LEO) based satellite access through link level simulations has been identified as one of the radio-layer-1 objectives in [5]. These studies and releases by $3 \mathrm{GPP}$ for $5 \mathrm{G}$ development, adds to the relevance of research activities to integrate previously independent satellite and terrestrial networks.

The fifth generation wireless communication systems aim to support a wide variety of services and user requirements in a cost effective manner rendering high quality of service (QoS). Waveforms have been a critical and fundamental aspect in defining the wireless communication standards. The main driving factors put forward by the research community to meet the heterogeneous requirements of $5 \mathrm{G}$ are new candidate waveforms for NR using different numerologies for flexibly rendering the waveform parameters. The typical satellite channel impairments that impose serious challenges in the physical layer design to support NR operations via satellites are large propagation delay, high Doppler shift due to mobility, high non-linear degradation, power-limited link budget and phase noise. The limitations of classical single numerology OFDM such as large spectral leakage and high peak-to-average power ratio (PAPR) become more significant under the design challenges in this scenario.

Requirements such as more efficient spectrum utilization and stringent synchronization have motivated the investigation of frequency-localized waveforms over the last decade. Filtered-OFDM (f-OFDM) [6], [7], windowed-OFDM (WOFDM) [8], [9], universal filtered multi-carrier (UFMC) and [10], [11] preserve the transceiver structure of OFDM and have reduced out of band emission. Hence these are recommended by $3 \mathrm{GPP}$ as the waveforms for new-radio (NR) in order to preserve the advantages of OFDM as well as maintain backward compatibility. The applicability of OFDM and filter bank multi-carrier (FBMC) in the return link of an HTS communication system is studied in [12]. The performance assessment of 5G NR physical layer aspects for geostationary (GEO) satellite scenario is carried out in [13]. The adaptability of candidate waveforms under satellite channel impairments for single numerology scenario is evaluated in [14]. The NR air interface analysis for different user access types with transparent payload satellites was performed in [15]. The high power amplifier (HPA) on board of the satellite will induce considerable non-linear distortion in the multi-carrier signal. The non-linear distortion, multipath and Doppler effects become significant in the satellite to user link in the direct user access scenario with regenerative LEO satellite. The NR 
numerology and waveform evaluation for this scenario has not been previously studied to the best of our knowledge. PAPR reduction methods that have smaller changes to NR radio protocols need to be considered when NR is adapted for NTN and hence DFT-spread-f-OFDM (DFT-s-f-OFDM) and DFT-spread-W-OFDM (DFT-s-W-OFDM) which have lower PAPR than their counterparts, are also analysed.

In the framework of 3GPP standardisation for satelliteterrestrial integration, based on the Radio Access Network (RAN) activities and critical decisions on the physical and MAC layers for the NR air interface, this paper reviews the potential areas of impact on NR from a physical layer perspective. We analyse the NR waveforms with different numerologies according to the guidelines set by 3GPP regarding the key LEO satellite parameters and simulation assumptions for the integrated scenario. The remainder of the paper is organized as follows. A brief overview on satellite-terrestrial channel model is presented in Section II. In section III, the non-linearity and PAPR issues are outlined. The Doppler shift compensation and sub-carrier bandwidth adaptation are discussed in Section IV. The numerical analysis and simulation results of candidate waveforms for different numerologies and satellite scenario are presented in Section $\mathrm{V}$ and conclusions are drawn in section VI.

\section{SySTEM ARCHITECTURE}

A brief overview of the integrated satellite terrestrial architecture is provided in this section. The different architecture options for the integrated satellite terrestrial solutions can be categorised on the basis of the access type as direct or via an on ground relay node, and the payload type as transparent or regenerative [15]. A generic block diagram of the integrated satellite terrestrial architecture, is shown in Fig. 1. Unlike the transparent bent-pipe payload scenario, in a regenerative satellite, the on-board processor enables base station functions like demodulation/decoding, switch and/or routing, coding/modulation along with filtering, frequency translation and amplification. The regenerative architecture helps to reduce the prorogation delays for physical layer procedures but at the expense of high complexity and cost. Hence, power efficient transmission is critical in the regenerative scenario for direct user access, especially in the satellite to user link, where NR air interface should be employed.

The OFDM based NR air interface waveforms can generally be represented as [14]

$$
s(n)=\sum_{k=-\infty}^{+\infty} \sum_{m=0}^{M} d_{k, m} p(n-k M) e^{(j 2 \pi m F(n-k M))}
$$

where $d_{k, m}$ represents the actual data symbols or DFT spread version of the data symbols, modulated at $k$-th symbol index and $m$-th sub-carrier index, $p(n)$ denotes the prototype filter and $F$ is the sub-carrier spacing. In W-OFDM, the persub-carrier filtering with $p(n)$ is effectively carried out by time-domain windowing using a pulse with smooth edges in time domain and subsequently sharp decay in frequency



Fig. 1: Generic block diagram of integrated satellite terrestrial architecture. The transparent and regenerative payload links are shown as dotted and solid lines respectively.

domain. For f-OFDM, the conventional CP-OFDM signal is first synthesised using IFFT operation with $p(n)$ as rectangular pulse and then inserting cyclic prefix. The f-OFDM signal $y(n)$, is then generated by filtering the CP-OFDM signal through a band-pass filter with impulse response, $f(n)$, to obtain $y(n)=s(n) * f(n)$ where $*$ denotes linear convolution. The choice of transmit/receive window and filter functions, receiver processing and detailed implementation aspects are provided in [6], [8], [14].

\section{Power CONSTRAined LINK BUDGET}

A major design constraint in providing ubiquitous coverage under high propagation path loss in the integrated-satelliteterrestrial scenario is to maximize power efficiency and throughput in forward and reverse links. The HPA on board the satellite or at the UE has to be operated at a point closest to saturation point where the input-output relationship is highly non-linear. Waveforms with high PAPR will be operated in the non-linear region of HPA, which results in amplitude and phase distortions as well as out-of-band emission.

This demands backing off the input power to the HPA so that the operating point is in the non-linear region, which results in reduced transmit power and eventually fails to guarantee sufficient SNR. Hence, new air interface waveforms must feature low PAPR and must be more robust against non-linear distortions. Signal processing for PAPR reduction and pre-distortion must be developed for operating the HPA with the minimum output back-off. The regenerative payload architecture demands on-board-processing of the signals where the pre-distortion of the signals can be performed in the digital domain and then converted to analog by DAC which is fed to the HPA. The magnitude of the digital signals are changed as a result of the pre-distortion operation and hence may be subject to clipping by the DAC, depending on the dynamic range of DAC and the maximum signal amplitude after predistortion. Fig. 2 represents the combined amplitude/amplitude 




Fig. 2: Combined HPA-DAC non-linear amplitude transformation function model

(AM/AM) characteristics of HPA and DAC. The saturation point and the clipping point of DAC is assumed to be at 0 $\mathrm{dB}$. The amplitude characteristics can be divided into three regions. The low power efficiency linear region of HPA, nonlinear high power efficiency region of HPA and the constant amplitude region due to clipping by DAC. The output $v$ of HPA to an input signal $u=a e^{j \theta}$, can be expressed as

$$
v=T(a) e^{j((\theta)+\phi(a))}
$$

where $T(a)$ and $\phi(a)$ are the AM/AM and amplitude/phase (AM/PM) input-output characteristic functions of the HPA respectively. The phase distortion, $\phi(a)$ can be pre-calculated and subtracted from the original phase of the signal before input to the HPA so that phase distortion is cancelled out. The amplitude distortion can be corrected using inverse function based pre-distortion method such that $T\left(T^{-1}(a)\right)=a$. The non-linear distortions due to clipping cannot be corrected using pre-distortion operation.

\section{DOPPLER SHIFT COMPENSATION AND SUb-CARRIER BANDWIDTH ADAPTATION}

The Doppler effects in the non geostationary satellite based integrated-satellite-terrestrial networks are higher than that in the terrestrial scenario due to the larger relative velocity between satellite and the user equipment which results in inter carrier interference (ICI). A worst case user velocity of $1000 \mathrm{~km} / \mathrm{h}$ and a low earth orbit (LEO) satellite in S band (2 GHz) correspond to a maximum Doppler shift of \pm 48 $\mathrm{kHz}$, which is much higher than the frequency error robustness requirement of NR standards (5 ppm, $10 \mathrm{kHz}$ for $\mathrm{S}$ band). Even though these Doppler shift values are not covered by current $5 \mathrm{G}$ specifications, based on the knowledge of the ephemeris of the satellite and user location, blind coarse frequency shift compensation can be employed to bring the residual Doppler shift to less than $5 \mathrm{ppm}$. The frequency offset can be perfectly compensated for the UE at beam centre but a residual frequency offset persists for the users that are away from the beam centre [16]. This residual CFO will be maximum at beam edges and depends on beam footprint diameter, the relative radial velocity of the satellite in relation to the beam center and user equipment. The maximal residual frequency error values due to both satellite and UE movement for different altitudes and beam footprint diameters are presented in [16]. This residual $\mathrm{CFO}$ can be further reduced by extending the sub-carrier bandwidth of the OFDM based signal [2].

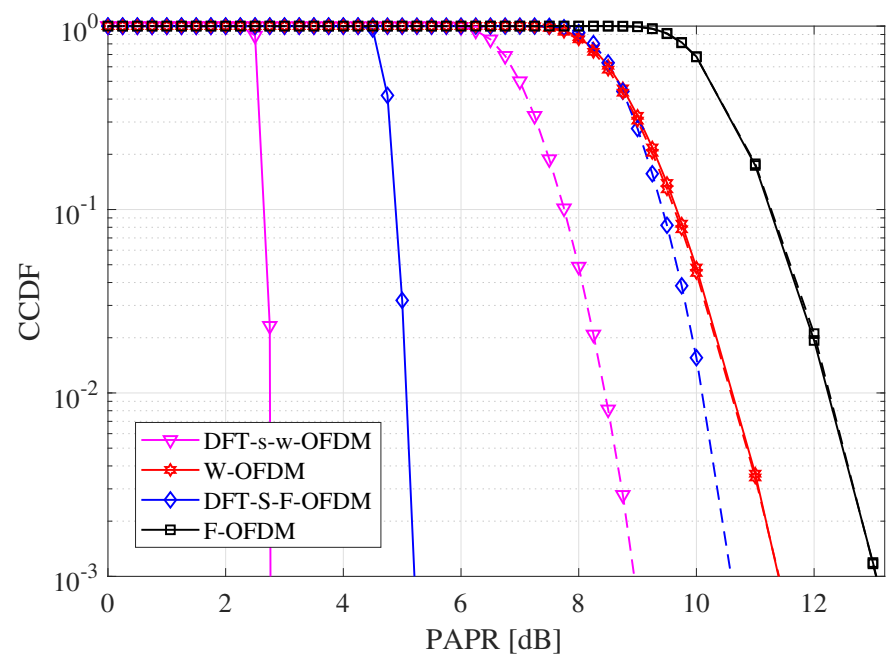

Fig. 3: PAPR comparison for candidate waveforms. The solid and dotted curves correspond to $N_{\text {used }}=N_{F F T}$ and $N_{\text {used }}=\frac{N_{F F T}}{2}$ respectively.

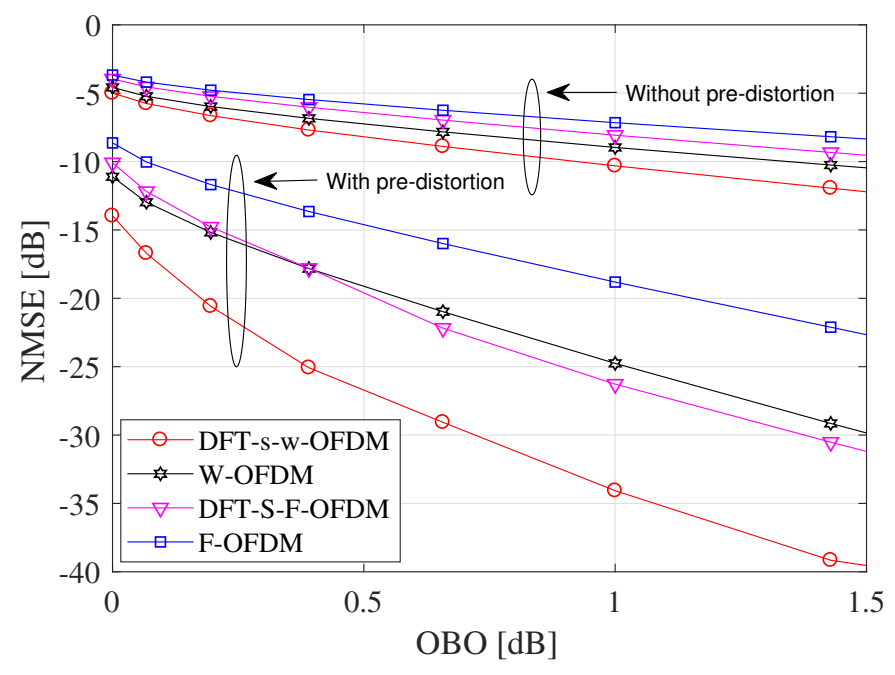

Fig. 4: NMSE versus OBO

\section{Simulation Results AND Discussion}

The numerical analysis of the 5G NR candidate waveforms and the impact of different numerologies in integrated satellite mobile communication scenario is presented in this section. Complementary cumulative distribution function (CCDF) versus PAPR comparison of the candidate waveforms for the number of used sub-carriers, $N_{\text {used }}=N_{F F T}$ and $N_{\text {used }}=$ $\frac{N_{F F T}}{2}$ are presented in Fig. 3 . 


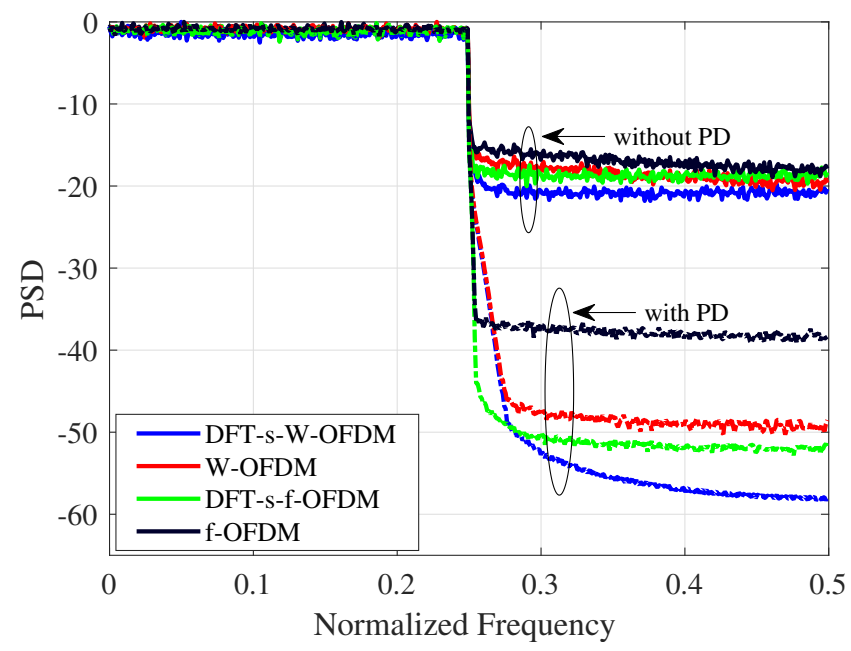

(a) $\mathrm{OBO}=3 \mathrm{~dB}, \mu=0$

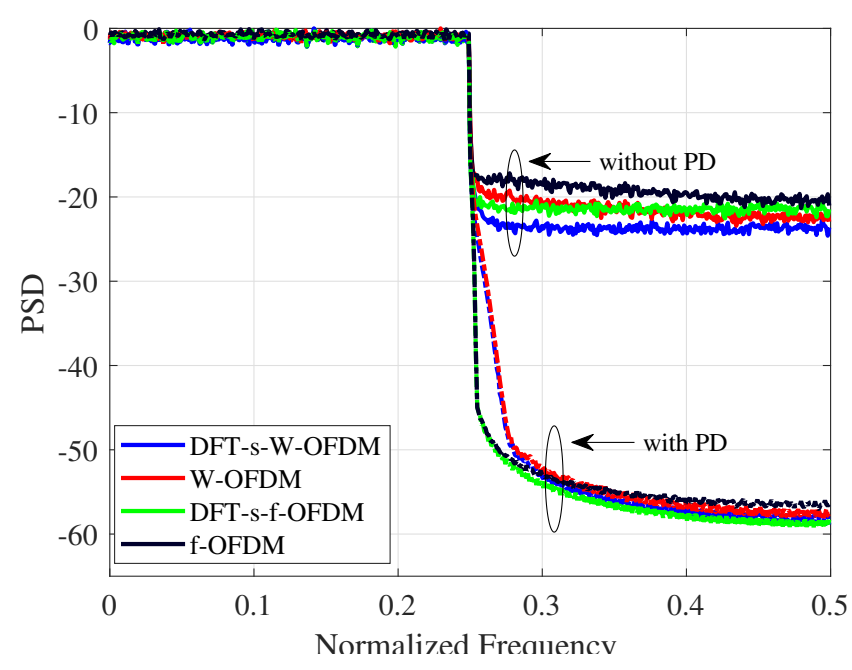

(c) $\mathrm{OBO}=5 \mathrm{~dB}, \mu=0$

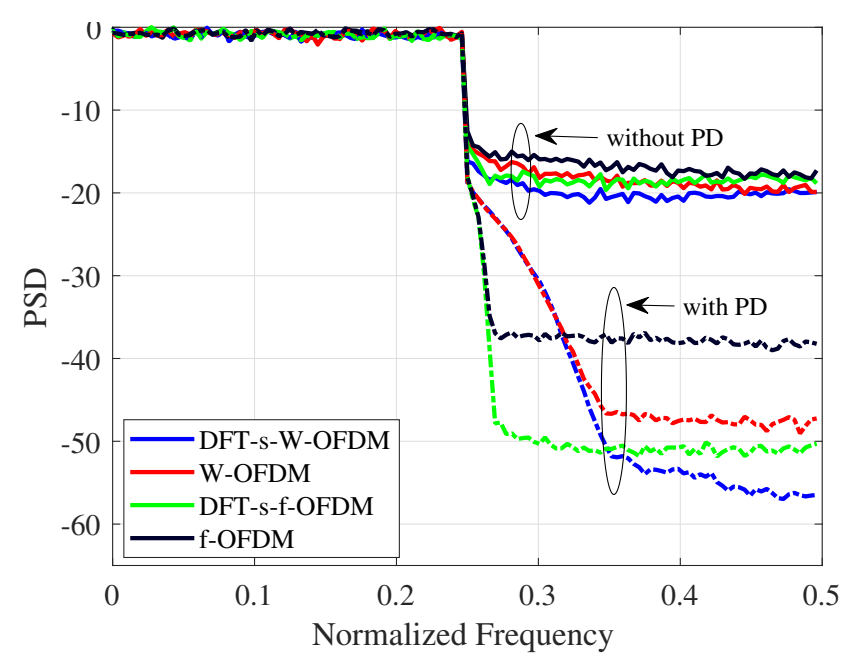

(b) $\mathrm{OBO}=3 \mathrm{~dB}, \mu=2$

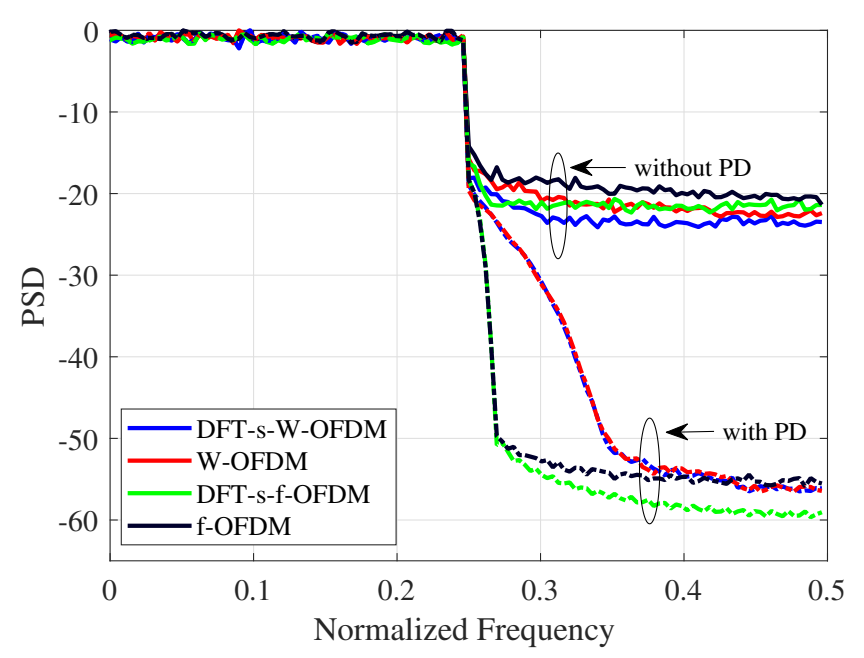

(d) $\mathrm{OBO}=5 \mathrm{~dB}, \mu=2$

Fig. 5: Power spectral density (PSD) comparison of waveforms for different OBO and numerologies. PSDs of waveforms with pre-distortion (PD) are shown using dotted lines

The fully loaded DFT spread waveforms corresponding to $N_{\text {used }}=N_{F F T}$ are equivalent to single carrier systems and hence have lower PAPR than that corresponding to $N_{\text {used }}=$ $\frac{N_{F F T}}{2}$. The DFT-s-W-OFDM and DFT-s-f-OFDM have lower PAPR due to DFT spreading. The windowed OFDM based waveforms have lower PAPR than the filtered waveforms.

The in-band distortion of the waveforms due to HPA is assessed using normalized mean square error (NMSE) performance. NMSE is defined as [17]

$$
\operatorname{NMSE}(\mathrm{dB})=10 \log \mathbb{E}\left\{\frac{\sum_{n=0}^{N_{\text {used }}-1}\left|d_{n}-\hat{d}_{n}\right|^{2}}{\sum_{n=0}^{N_{\text {used }}-1}\left|d_{n}\right|^{2}}\right\}
$$

where $d_{n}$ and $\hat{d}_{n}$ denote the transmitted and demodulated sym- bol respectively. The amplitude and phase input-output characteristics of a typical satellite TWTA provided by European Space Agency is used to introduce non-linear distortion in the present simulation. The AM/PM characteristics is modelled according to generic memoryless modified Saleh model [18] which uses 6 parameters. According to the model, $T_{\phi}(a)$ is defined as

$$
T_{\phi}(a)=\frac{\alpha a^{\eta}}{\left(1+\beta a^{\gamma}\right)^{\nu}}+\epsilon
$$

Amplitude pre-distortion is performed using the inverse function of a simplified model with fewer parameters derived from the 6 parameter model, which is given as

$$
T^{-1}(b)=\exp \left(\frac{1}{\eta} \ln \left(\frac{(b-\epsilon)}{\alpha-\beta(b-\epsilon)}\right)\right)
$$




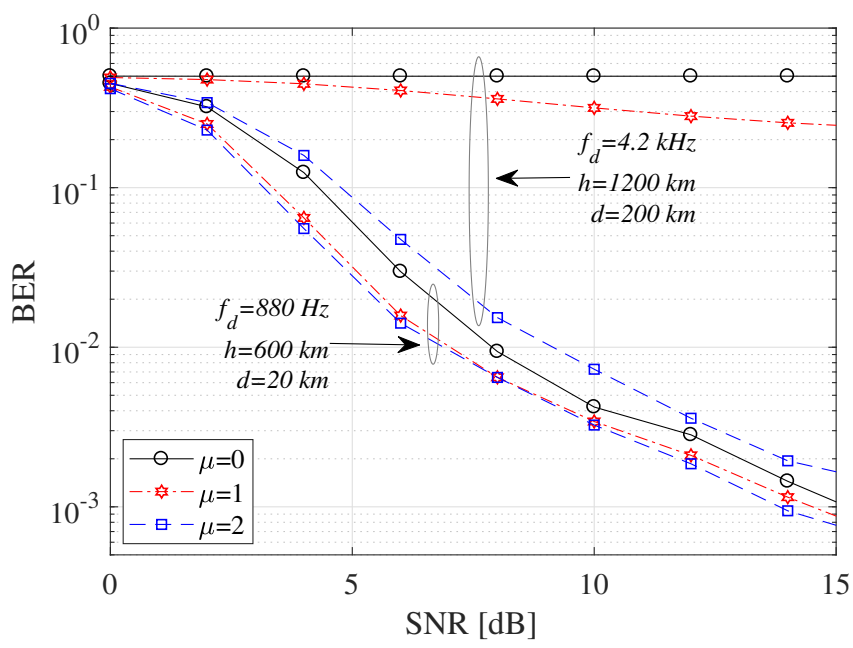

(a) W-OFDM

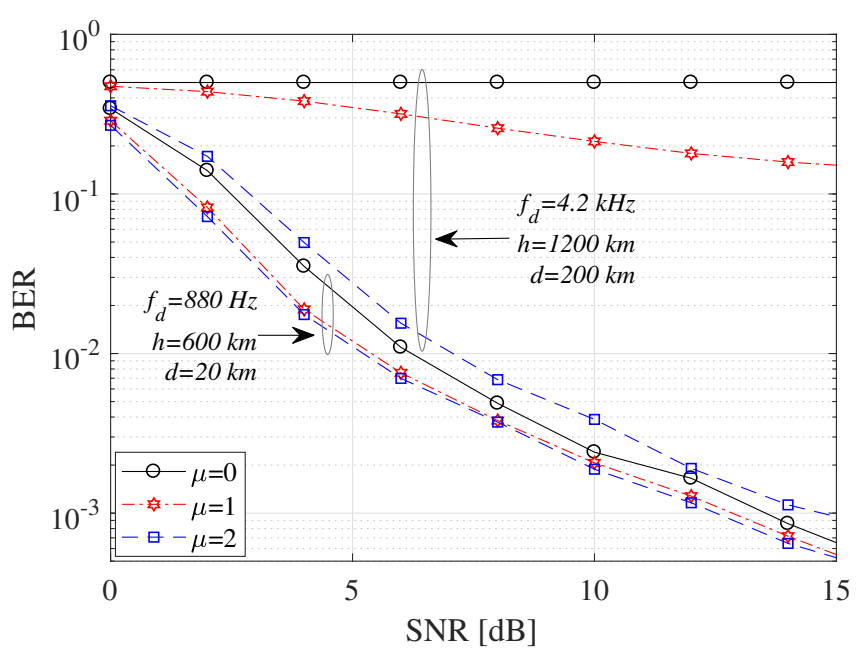

(c) f-OFDM



(b) DFT-s-W-OFDM

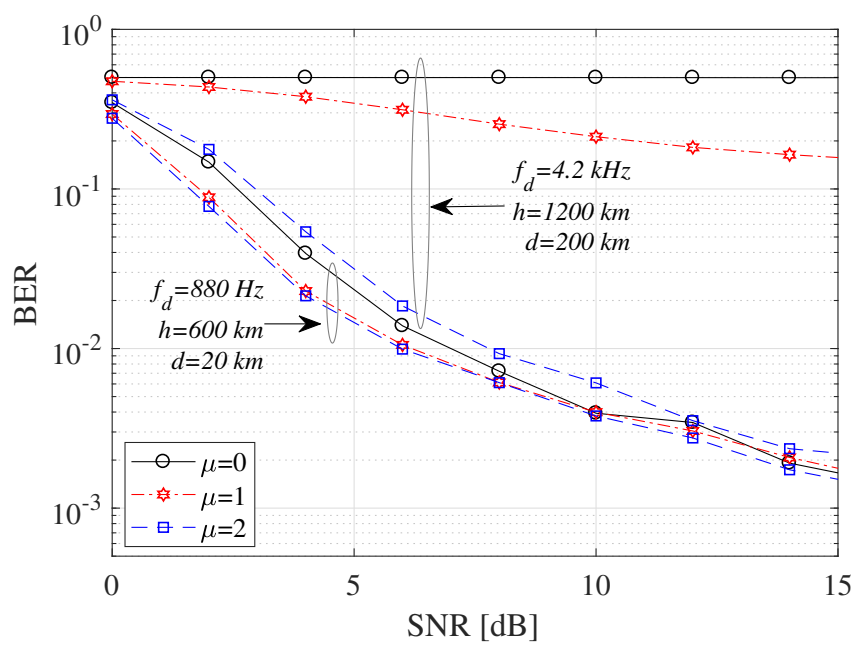

(d) DFT-s-f-OFDM

Fig. 6: BER versus $\mathrm{SNR}$ performance at $\mathrm{S}$ band $\mathrm{OBO}=3 \mathrm{~dB}$, maximum user velocity, $v=1000 \mathrm{~km} / \mathrm{h}$, Elevation Angle $=10^{\circ}$, Delay spread=10 ns.

The parameters of the HPA model for the AM/PM characteristics used in the simulation corresponding to (4) are obtained using curve fitting toolbox of MATLAB and which are $\alpha=4.107, \beta=5.203, \gamma=1.949, \eta=2.244$, $\mu=0.9249$ and $\epsilon=-0.02394$. The parameters of the inverse model for AM/AM characteristic (5) are $\alpha=11.38, \beta=$ $11.95, \gamma 1=0.949, \eta=2.058$ and $\epsilon=0.1385$. The NMSE versus OBO comparison of the candidate waveforms for the basic numerology with and without PD are reported in Fig. 4. The NMSE of the waveforms are reduced with pre-distortion operation and DFT-s-W-OFDM has the lowest NMSE and inband distortion compared to the other waveforms.

The out of band emission (OOBE) of the waveforms are analysed using the power spectral density (PSD) plots under HPA non-linearity and are compared for different OBO values and numerologies in Fig. 5. The subcarrier spacing corresponding to each numerology $\mu$ is $2^{\mu} \times 15 \mathrm{kHz}$
In-order to investigate the effect of sub-carrier bandwidth variation on residual Doppler shift, the BER versus SNR performance comparison of the candidate waveforms for different LEO satellite scenario and numerologies under the effect of non-line-of-sight (N-LOS) multipath fading, Doppler shift, phase noise and HPA non-linearity at $\mathrm{OBO}=3 \mathrm{~dB}$, is presented in Fig. 6. Two different LEO satellite scenario are considered for each waveform, corresponding to maximum user equipment velocity of $1000 \mathrm{~km} / \mathrm{h}$. The tapped delay line (TDL) channel model recommended by 3GPP [2] for non-line-of-sight satellite to user link in a terrestrial-NTN integrated scenario in sub-urban environment is used in the simulations. The TDL model is used to generate the impulse response corresponding to the multipath channel with Rayleigh fading coefficients at satellite elevation angle (EA) of $10^{\circ}$ and delay spread of $10 \mathrm{~ns}$. Half-rate convolutional coding is used for channel coding and one-tap zero-forcing equalization 


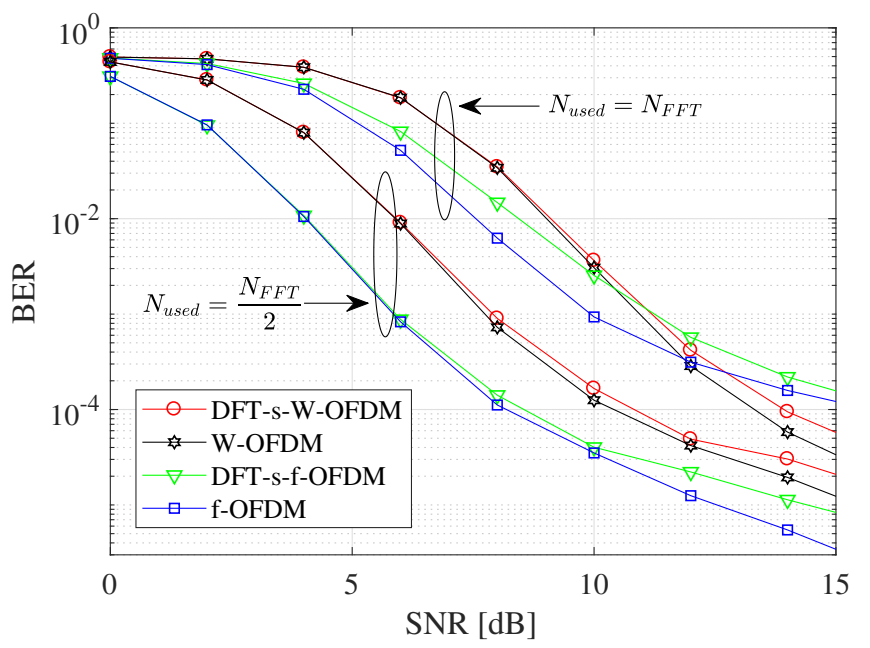

(a) Delay spread $=0, \mu=0$



(b) Delay spread $=10 \mathrm{~ns}, \mu=2$

Fig. 7: BER versus SNR comparison of waveforms at $\mathrm{S}$ band, $\mathrm{OBO}=3 \mathrm{~dB}$, LEO Satellite altitude $=600 \mathrm{~km}$, Elevation Angle $=10^{\circ}$, Beam footprint diameter, $\mathrm{d}=20 \mathrm{~km}$.

employed. The parameters of the S-band satellite phase-noise mask in [19] is used for the simulation of phase noise. The residual carrier frequency offset (CFO) values corresponding to $\mathrm{S}$ band are calculated and normalized using the sub-carrier bandwidths of the NR numerologies for different LEO satellite scenarios in [16] which are presented in Table I. Here, $\epsilon$ is the normalized CFO, $f_{d}$ represents maximum residual Doppler shift, $v$ is the user equipment velocity, $h$ and $d$ denote the LEO satellite altitude and beam foot print diameter respectively.

TABLE I: Residual CFO, $\epsilon$ for different numerologies for $\mathrm{S}$ band

\begin{tabular}{|c|c|c|c|c|c|c|}
\hline \multirow{2}{*}{$\begin{array}{c}h \\
(\mathrm{~km})\end{array}$} & \multirow{2}{*}{$\begin{array}{c}d \\
(\mathrm{~km})\end{array}$} & \multirow{2}{*}{$\begin{array}{c}v \\
(\mathrm{~km} / \mathrm{h})\end{array}$} & \multirow{2}{*}{$\begin{array}{c}f_{d} \\
(\mathrm{ppm})\end{array}$} & $\begin{array}{c}\text { Num-0 } \\
15 \mathrm{kHz}\end{array}$ & $\begin{array}{c}\text { Num-1 } \\
30 \mathrm{kHz}\end{array}$ & $\begin{array}{c}\text { Num-2 } \\
60 \mathrm{kHz}\end{array}$ \\
\cline { 5 - 7 } & & & $\epsilon$ & $\epsilon$ & $\epsilon$ \\
\hline \multirow{3}{*}{600} & \multirow{2}{*}{200} & 1000 & 4.3 & 0.573 & 0.286 & 0.143 \\
\cline { 3 - 7 } & & 0 & 4.14 & 0.552 & 0.276 & 0.138 \\
\cline { 3 - 7 } & \multirow{2}{*}{20} & 1000 & 0.44 & 0.058 & 0.029 & 0.014 \\
\cline { 3 - 7 } & \multirow{2}{*}{200} & 0 & 0.42 & 0.056 & 0.028 & 0.014 \\
\cline { 3 - 7 } & \multirow{2}{*}{40} & 1000 & 2.1 & 0.280 & 0.140 & 0.070 \\
\cline { 3 - 7 } & & 0 & 2.01 & 0.268 & 0.134 & 0.067 \\
\cline { 3 - 7 } & & 0 & 0.42 & 0.056 & 0.028 & 0.014 \\
\hline
\end{tabular}

The maximum residual Doppler shift when LEO satellite altitude, $h=1200 \mathrm{~km}$ and beam foot print diameter, $d=200$ $\mathrm{km}$ is $4.2 \mathrm{kHz}$ and that when $h=600 \mathrm{~km}$ and $d=20 \mathrm{~km}$ is $880 \mathrm{~Hz}$. The reduction in BER with the increase in sub-carrier bandwidth from $15 \mathrm{kHz}(\mu=0)$ to $60 \mathrm{kHz}(\mu=2)$ is illustrated in Fig. 6. This happens because, as the sub-carrier bandwidth increases, the inter carrier interference (ICI) produced due the Doppler shift decreases. For the LEO satellite scenario corresponding to $f_{d}=4.2 \mathrm{kHz}, h=1200 \mathrm{~km}$ and $d=200$ $\mathrm{km}$, it is evident that the BER is drastically reduced when numerology $\mu=2$ is chosen, for all the waveforms.

The BER versus SNR performance comparison of the candidate waveforms for different number of used sub-carriers at $\mathrm{OBO}=3 \mathrm{~dB}$, LEO satellite altitude, $h=600 \mathrm{~km}$, elevation angle of $10^{\circ}$, beam footprint diameter, $d=20 \mathrm{~km}$ and S-band phase noise are shown in Fig. 7. Fig. 7a and Fig. 7b shows the BER comparison plots corresponding to delay spread $=0$, $\mu=0$ and delay spread $=10 \mathrm{~ns}, \mu=2$ respectively. In Fig. 7a, it is observed that, when the number of used subcarriers, $N_{\text {used }}=\frac{N_{F F T}}{2}$, the filtered OFDM based waveforms show better BER performance than the windowed OFDM based waveforms when delay spread is zero. For fully loaded OFDM systems, i.e. $N_{\text {used }}=N_{F F T}$, the windowed OFDM based waveforms show better BER performance at high SNR points. In the presence of multipath delay spread, residual Doppler shift and phase noise, if pre-distortion is employed, f-OFDM provides the best BER performance among the other considered waveform candidates. DFT-s-f-OFDM incur significant performance losses compared to other waveforms in the high SNR region. It is also worth noting that, f-OFDM and W-OFDM have better robustness against the considered channel impairments than the DFT spread counterparts despite the large PAPR.

\section{CONCLUSION}

In this paper, the performance of 5G NR numerologies and the candidate waveforms are analysed using key satellite parameters and simulation assumptions recommended by $3 \mathrm{GPP}$ in the context of RAN1 objectives associated with solutions for NR to support NTN. The change in NR numerology has significant effect on the BER performance in the integrated satellite terrestrial scenario as the large Doppler for LEO satellites can be accommodated by increasing the sub-carrier bandwidth. Even though increasing sub-carrier bandwidth results in a lower CP length, simulation results indicate that, it is sufficient to protect the signals against multipath interference. Even though the DFT spread waveforms with minimum PAPR and OOBE are robust against non-linear distortion due to HPA, simulation results confirm that, with appropriate numerology, 
pre-distortion and channel equalization, the W-OFDM and fOFDM waveforms show improved performance in the presence of high residual Doppler shift, non-linear distortion, NTN-multipath channel and phase noise. Considering all the analysis and observations, despite the cost of increased complexity, filtering is better than windowing and f-OFDM can be recommended as the most promising candidate waveform for the satellite to user link in a regenerative LEO satellite scenario for direct user access. Quantification of the trade-off between non-linear distortion and ICI due to Doppler becomes significant to select the best waveform and numerology for high power transmission, which can be considered for further studies.

\section{ACKNOWLEDGMENT}

This work was sponsored by China Academy of Space Technology (CAST)-Xian. The authors also would like to acknowledge the support of the University of Surrey 5GIC (http://www.surrey.ac.uk/5gic).

\section{REFERENCES}

[1] 3GPP, "Study on Scenarios and Requirements for Next Generation Access Technologies," 3rd Generation Partnership Project (3GPP), Technical Report (TR) 38.821, 05 2017, version 0.6.0.

[2] — " "Study on New Radio (NR) to support non-terrestrial networks," 3rd Generation Partnership Project (3GPP), Technical Report (TR) RAN-80, 08 2018, version 15.0.0.

[3] _ " "Technical Specification Group Radio Access Network NR Physical channels and modulation," 3rd Generation Partnership Project (3GPP), Technical Specification (TS) TS 38.211, 04 2019, version 14.2.0.

[4] — " "Preliminary views on NTN activities," 3rd Generation Partnership Project (3GPP), Discussion RAN83, 03 2019, RP-190130.

[5] — - "Revised SID: Study on solutions for NR to support non-terrestrial networks," 3rd Generation Partnership Project (3GPP), SID revised-97 RAN-82, 12 2019, RP-182444.

[6] J. Abdoli, M. Jia, and J. Ma, "Filtered OFDM: A new waveform for future wireless systems," in 2015 IEEE 16th International Workshop on Signal Processing Advances in Wireless Communications (SPAWC), June 2015, pp. 66-70.
[7] L. Zhang, A. Ijaz, P. Xiao, M. M. Molu, and R. Tafazolli, "Filtered OFDM systems, algorithms, and performance analysis for $5 \mathrm{G}$ and beyond," IEEE Transactions on Communications, vol. 66, no. 3, pp. 1205-1218, March 2018.

[8] R. Zayani, Y. Medjahdi, H. Shaiek, and D. Roviras, "WOLA-OFDM: A potential candidate for asynchronous 5G," in 2016 IEEE Globecom Workshops (GC Wkshps), Dec 2016, pp. 1-5.

[9] X. Zhang, L. Zhang, P. Xiao, D. Ma, J. Wei, and Y. Xin, "Mixed numerologies interference analysis and inter-numerology interference cancellation for windowed OFDM systems," IEEE Transactions on Vehicular Technology, vol. 67, no. 8, pp. 7047-7061, Aug 2018.

[10] V. Vakilian, T. Wild, F. Schaich, S. ten Brink, and J. Frigon, "Universalfiltered multi-carrier technique for wireless systems beyond LTE," in 2013 IEEE Globecom Workshops (GC Wkshps), Dec 2013, pp. 223228.

[11] L. Zhang, A. Ijaz, P. Xiao, A. Quddus, and R. Tafazolli, "Subband filtered multi-carrier systems for multi-service wireless communications,' IEEE Transactions on Wireless Communications, vol. 16, no. 3, pp. 1893-1907, March 2017.

[12] S. Dimitrov, N. Privitera, R. Suffritti, G. Boccolini, A. B. Awoseyila, and B. G. Evans, "Spectrally efficient waveforms for the return link in satellite communication systems," in 2015 European Conference on Networks and Communications (EuCNC), June 2015, pp. 6-10.

[13] N. Cassiau, L. Maret, J. Doré, V. Savin, and D. Kténas, "Assessment of 5G NR physical layer for future satellite networks," in 2018 IEEE Global Conference on Signal and Information Processing (GlobalSIP), Nov 2018, pp. 1020-1024.

[14] A. Jayaprakash, H. Chen, P. Xiao, B. G. Evans, Y. Zhang, J. Y. Li, and A. B. Awoseyila, "Analysis of candidate waveforms for integrated satellite-terrestrial 5G systems," in 2019 IEEE 2nd 5G World Forum (5GWF), Sep. 2019, pp. 636-641.

[15] A. Guidotti, A. Vanelli-Coralli, M. Conti, S. Andrenacci, S. Chatzinotas, N. Maturo, B. Evans, A. Awoseyila, A. Ugolini, T. Foggi, L. Gaudio, N. Alagha, and S. Cioni, "Architectures and key technical challenges for 5G systems incorporating satellites," IEEE Transactions on Vehicular Technology, vol. 68, no. 3, pp. 2624-2639, March 2019.

[16] 3GPP, "On Doppler shift compensation and Timing Advance in NTN," 3rd Generation Partnership Project (3GPP), Discussion RAN1-97, 05 2019, R1-1907390.

[17] B. F. Beidas, "Adaptive digital signal predistortion for nonlinear communication systems using successive methods," IEEE Transactions on Communications, vol. 64, no. 5, pp. 2166-2175, May 2016.

[18] M. O'droma, S. Meza, and Y. Lei, "New modified Saleh models for memoryless nonlinear power amplifier behavioural modelling," IEEE Communications Letters, vol. 13, no. 6, pp. 399-401, 2009.

[19] 3GPP, "Key satellite parameters and simulation assumptions for NTN," 3rd Generation Partnership Project (3GPP), Discussion RAN1-96, 04 2019, R1-1905216. 